\title{
Tracheostomy during COVID-19 pandemic - a novel approach
}

\author{
Velda Ling Yu Chow MD ${ }^{1}$ \\ ${ }^{1}$ University of Hong Kong Li Ka Shing Faculty of Medicine
}

April 28, 2020

\begin{abstract}
Background This study describes a novel approach in reducing SARS-CoV-2 transmission during tracheostomy. Methods Five patients underwent tracheostomy between 01 April 2020 and 17 April 2020. A clear and sterile plastic drape was used as an additional physical barrier against droplets and aerosols. Operative diagnosis; droplet count and distribution on plastic sheet and face shields were documented. Results Tracheostomy was performed for patients with carcinoma of tonsil ( $\mathrm{n}=2$ ) and nasopharynx $(n=1)$, and aspiration pneumonia $(n=2)$. Droplet contamination was noted on all plastic sheets $(n=5)$. Droplet contamination was most severe over the central surface at $91.5 \%$ (86.7\%-100.0\%) followed by the left and right lateral surfaces at $5.2 \%(6.7 \%-10.0 \%)$ and $3.3 \%(6.7 \%-10.0 \%)$ respectively. No droplet contamination was noted on all face shields. Conclusion Plastic drapes can help reduce viral transmission to health care providers during tracheostomy. Face shields may be spared which in turn helps to conserve resources during the COVID-19 pandemic.
\end{abstract}

\section{AUTHORS}

Velda Ling Yu Chow MD, MS 1

Jimmy Yu Wai Chan MD, MS, PhD 1

Valerie Wai Yee Ho MD 1

Sherby Suet Ying Pang MD 1

George Chung Ching Lee MD, BDS 1

Melody Man Kuen Wong MD1

Arthur Shing Ho Lo MD 1

Frances Lui MD 2

Clara Ching Mei Poon MD 2

Stanley Thian Sze Wong BSc, PhD 1

CORRESPONDING AUTHOR

Velda Ling Yu Chow MD, MS

INSTITUION

Division of Head and Neck Surgery

Department of Surgery

University of Hong Kong Li Ka Shing Faculty of Medicine

Queen Mary Hospital 
Department of Anesthesiology

University of Hong Kong Li Ka Shing Faculty of Medicine Queen Mary Hospital

ADDRESS FOR CORRESPONDANCE AND REPRINT

Division of Head and Neck Surgery

Department of Surgery

University of Hong Kong Li Ka Shing Faculty of Medicine

Queen Mary Hospital

102 Pokfulam Road,

Hong Kong SAR, China

Telephone: +85222552208

Fax: +85228193780

E-mail: vlychow@gmail.com

\section{DISCLOSURE}

No financial disclosure. This paper is not based on previous communication to a society or meeting

\section{RUNNING TITLE}

Tracheostomy during COVID-19 - a novel approach

\section{KEY WORDS}

Tracheostomy, COVID-19, Novel approach, Conservation of PPE, Head and neck cancer

\section{ABSTRACT}

\section{Background}

This study describes a novel approach in reducing SARS-CoV-2 transmission during tracheostomy.

\section{Methods}

Five patients underwent tracheostomy between 01 April 2020 and 17 April 2020. A clear and sterile plastic drape was used as an additional physical barrier against droplets and aerosols. Operative diagnosis; droplet count and distribution on plastic sheet and face shields were documented.

\section{Results}

Tracheostomy was performed for patients with carcinoma of tonsil $(n=2)$ and nasopharynx $(n=1)$, and aspiration pneumonia $(n=2)$. Droplet contamination was noted on all plastic sheets $(n=5)$. Droplet contamination was most severe over the central surface at $91.5 \%$ (86.7\%-100.0\%) followed by the left and right lateral surfaces at $5.2 \%(6.7 \%-10.0 \%)$ and $3.3 \%(6.7 \%-10.0 \%)$ respectively. No droplet contamination was noted on all face shields.

\section{Conclusion}

Plastic drapes can help reduce viral transmission to health care providers during tracheostomy. Face shields may be spared which in turn helps to conserve resources during the COVID-19 pandemic.

\section{INTRODUCTION}


The novel coronavirus disease 2019 (COVID-19) is caused by SARS-CoV-2 virus. SARS-CoV-2 is found in high abundance in the upper aerodigestive tract mucosa [1]. It is known to be transmitted via close contact, droplet and aerosols from aerosol generating procedures (AGP) such as tracheostomy [2].

COVID-19 is associated with acute respiratory distress syndrome that requires patients to be intubated and may become dependent on mechanical ventilation. Patients with prolonged ventilation may require tracheostomy to optimise weaning from ventilatory support. [3] In Queen Mary Hospital, an experienced head and neck surgeon will be summoned to perform tracheostomy on such patients.

As head and neck surgeons, we are constantly exposed when resecting tumours arising from mucosa in the upper aerodigestive tract, in addition to tracheostomy and laryngectomy. Our patients may be asymptomatic at the time of presentation, and there is currently no accurate way of COVID-19 diagnosis. [4-5] Hence, we are at particular risk of becoming infected when performing tracheostomy during the COVID-19 pandemic.

World Health Organisation (WHO), Centres for Disease Control and Prevention (CDC) and Centre for Health Protection (CHP) recommend full barrier protection when performing AGP for unknown, suspected and confirmed COVID-19 patients in order to avoid disease transmission to health care providers. Such personal protective equipment (PPE) includes gloves, goggles, face shield and gowns, as well as items filtering facepiece respirators such as N95 or powered air-purifying respirator (PAPR) hoods and aprons [6-8].

The number of confirmed COVID-19 cases has soared since its first description in. December 2019 - as of 18 April 2020, there are 2121675 confirmed cases worldwide, of which Hong Kong accounts for 1024. [9,10] Such an escalation in the number of infected has resulted in a global shortage of PPE.

This study describes a novel approach which aims to decrease viral transmission when performing tracheostomy during the COVID-19 pandemic and at times of PPE shortage.

\section{MATERIALS AND METHODS}

All patients who underwent tracheostomy in the Division of Head and Neck Surgery of the Department of Surgery, The University of Hong Kong at Queen Mary Hospital and Gleneagles Hong Kong Hospital between 01 April 2020 and 17 April 2020 were included.

All operations were performed by a consultant surgeon accompanied by 1 scrub nurse and 1 consultant anaesthetist. Full barrier protection was adopted by all three parties. Intubation under general anaesthesia was performed by anaesthetist. Two horizontal anaesthetic screen supports were then placed and secured with universal rotary clamps on patient's left bedside: 1 anaesthetic screen support was placed at head level making sure not to limit the anaesthetist's view and working space; the other was placed at the level of patient's umbilicus at a height of $20 \mathrm{~cm}$ from patient's truncal surface. (Figure 1) The lower anaesthetic screen could be placed further apart and set at a greater height to ensure adequate working space for the operating surgeon. Skin was prepared and draped with disposable surgical drapes (3M Hong Kong) in the usual manner for tracheostomy, exposing the inferior border of mandible, bilateral neck and sternal angle. The 2 anaesthetic screen supports were covered by surgical drapes.

A clear and sterile plastic sheet measuring $120 \mathrm{~cm} \mathrm{x} 140 \mathrm{~cm}$ was placed over the operating field. The sheet was then pulled taut and secured over the operating field using sterile clips for mounting on the 2 horizontal anaesthetic screens. The caudal and left lateral edge of the plastic sheet was sealed using adhesive $3 \mathrm{M}$ tape. The cranial end of the sterile drape was not taped to allow manipulation of endo-tracheal tube by anaesthetist. The right side of the plastic sheet was not taped to allow the surgeon to operate from beneath. (Figure 2) A $1 \mathrm{~cm}$ puncture was made over the left upper corner of the central operating field for placement of smoke evacuation suction tubing. The hole was sealed and tubing secured with Tegaderm (3M Hong Kong). Suction for smoke evacuation was only used during tissue dissection with monopolar diathermy prior to tracheotomy. (Figure. 3)

Scrub nurse was positioned opposite the surgeon's right hand. Tracheostomy was performed as described by Wei, ensuring good communication with our anaesthetic colleague throughout the operation. [11] Skin 
incision was performed with scalpel knife, followed by soft tissue dissection with monopolar diathermy. Tracheotomy was performed with a scalpel knife after securing haemostasis and all suction devices switched off. After insertion of a cuffed Portex tracheostomy tube of appropriate size, the cuff was inflated. The tracheostomy tube was connected to a ventilator tubing which was passed under the plastic sheet and sterile drapes on the side of ventilator. Ventilation was resumed by the anaesthetist once closed ventilation circuit was secured. Tracheostomy tube was secured with 4 stitches using $3 / 0$ Nylon once successful ventilation was confirmed.

On completion of tracheostomy, the central and bilateral surfaces of the plastic sheet were marked with $7 \mathrm{~cm} \mathrm{x}$ $7 \mathrm{~cm}$ grids. (Figure 4) (Table 1). The face shield of surgeon and scrub nurse was removed after tracheostomy. The face shield used was a piece of optically clear, latex free plastic film measuring $32 \mathrm{~cm}$ in length and $22 \mathrm{~cm}$ in width with foam forehead cushion and elastic strap (A R Medicom Inc (Asia) Ltd.). It covered a full face length from forehead to neck, with outer edges of the face shield reaching bilateral ears. It had anti-fog and anti-glare properties with no hearing restrictions. Each face shield was put against a white background with 12 grids measuring $7 \mathrm{~cm} \times 7 \mathrm{~cm}$ each to facilitate counting at maximal magnification. Each plastic sheet was carefully removed and placed against a white background for counting.

The number and size of droplets splashed in each grid of the plastic sheet and face shield was counted using the surgical microscope Leica M720 0H5 (Leica Microsystems GmbH, Germany). The plastic sheets and face shields were discarded once counting was complete.

Operative diagnosis; operation duration; size, number and distribution of droplets on plastic shield and face shield for each party were documented.

\section{RESULTS}

Five patients with no clinical evidence of COVID-19 underwent tracheostomy: 2 patients who underwent radical tonsillectomy, modified radical neck dissection and free anterolateral thigh flap reconstruction for carcinoma of tonsil; 1 patient who underwent maxillary swing nasopharyngectomy for recurrent nasopharyngeal carcinoma; 2 patients who underwent tracheostomy for aspiration pneumonia and sputum retention. Average operation duration was $352.4(338.0-365.0)$ s. Droplet contamination was noted on all 5 plastic sheets. (Table 2) Droplet size ranged from $0.2 \mathrm{~mm}$ to $2.8 \mathrm{~mm}$. Droplet contamination was most severe over the central surface for all patients with an average of $91.5 \%(86.7 \%-100.0 \%)$ followed by the left lateral surface and right lateral surface at 5.2\% (6.7\%-10.0\%) and 3.3\% (6.7\%-10.0\%) respectively. (Table 3)

Droplet count contamination was mainly over the central upper half of plastic sheet overlying the site of operation in the lower neck. Total droplet count was highest along the centre-most column F at $25.9 \%$ $(\mathrm{n}=15)$. Droplet count decreased towards the periphery on both sides. The decline was greater on the left lateral surface at $10.3 \%(\mathrm{n}=6), 6.9 \%(\mathrm{n}=4), 3.4 \%(\mathrm{n}=2), 3.4 \%(\mathrm{n}=2), 1.7 \%(\mathrm{n}=1)$ and $0 \%$ along columns G, H, I J, K and L respectively compared with columns E, D, C, B and A at 17.2\% ( $\mathrm{n}=10), 24.1 \%(\mathrm{n}=14)$, $3.4 \%(\mathrm{n}=2), 1.7 \%(\mathrm{n}=1)$ and $1.7 \%(\mathrm{n}=1)$ respectively on the right lateral surface. (Table 3$)$

No droplet splash was documented on face shields of both the surgeon and scrub nurse for all patients.

\section{DISUCSSION}

SARS-CoV-2 is transmitted through close contact and droplets. Airborne transmission may occur during AGP including tracheal intubation, non-invasive ventilation, tracheotomy, cardiopulmonary resuscitation, manual ventilation before intubation, and bronchoscopy.

In view of the recent COVID-19 pandemic, tracheostomy guidelines and protocols have been revisited and updated with the aim of decreasing aerosol generation and viral transmission to health care providers. These include patient selection; timing of operation in relation to symptoms, quarantine duration and polymerase chain reaction test results; location of surgery; PPE requirements; minimising the number of health care providers; expertise in performing intubation and tracheostomy; and ways to decrease exposure to aerosolised secretions intra-operatively. [3,11-13] 
WHO, CDC and CHP advocates full barrier protection when performing AGP including a face shield which acts as an additional physical barrier against splashes, sprays, and spatter of body fluids. However, the use of face shield hinders the use of a head-light when performing head and neck surgery. Prolonged use can give rise to fogging, carbon dioxide retention especially when combined with respirator, and impaired communication. Furthermore, as the number of infected patients increases world-wide, there is a global shortage of PPE. As a result strategies have been formulated to optimise PPE availability include minimising the need for PPE in health care settings, and ensuring rational and appropriate use of PPE.

In this study, we proposed the use of 2 horizontal anaesthetic screens and a clear sterile plastic sheet draped over a tracheostomy operative field. The rationale is to create a spacious and sterile closed environment for the surgeon to work in whilst preventing droplet and aerosol escape during the procedure, ultimately reducing the chance of viral transmission. Such a set-up is readily available, functional, non-time-consuming and cost effective.

The 2 horizontal anaesthetic screens acted as struts. The height and distance of which could be adjusted by the surgeon to ensure adequate working space whilst not obstructing anaesthetist's view and working space at the cranial end. Surgical drapes were placed loosely over the 2 anaesthetic screens so that it conformed to the contour of the screens, resulting in a sterile and flat cranial and caudal surface, thereby increasing working space. Finally placement of a clear and sterile plastic sheet over the 2 anaesthetic screens and sealing over the caudal and left lateral edges helped to create a sterile box-like working area for the surgeon. It was imperative that the plastic sheet was pulled taut over the operative field so as not to compromise visibility. A long length of plastic sheet was allowed to drape over the cranial end without fixing to allow anaesthetist to reach the endo-tracheal tube. A length of plastic sheet measuring $14 \mathrm{~cm}$ over the right lateral surface acted as a hood against droplet and aerosol spillage, under which the surgeon's hands passed. Skin incision was performed using a scalpel knife followed by soft tissue dissection with monopolar diathermy. A suction catheter for smoke evacuation was placed over the surgeon's contralateral side to prevent fogging and impaired visibility. On reaching the anterior tracheal wall, haemostasis was secured. Suction was then turned off prior to tracheotomy. In order to minimise aerosol exposure, complete paralysis of the patient was ascertained throughout the procedure; mechanical ventilation was stopped prior to tracheotomy; suction was not used during and after tracheotomy; all tracheostomies were performed by consultant surgeons, consultant anaesthetists and scrub nurses experienced in the management of airways and the procedure. Such a setup did not adversely affect visibility and efficiency in performing tracheostomy as evidenced by an average operation duration of under 6 mins.

Our study demonstrated that despite meticulous tissue dissection and haemostasis, swift and bloodless tracheotomy, there was droplet contamination noted on plastic sheets of all 5 patients. Droplet contamination was centred over the lower neck which corresponded to the operating site for all patients. Droplet count decreased towards the periphery. The drop was less pronounced towards the right side where the surgeon stood and operated on. Droplet count was also noted on the right lateral surface of the plastic sheet, which acted as a hood further protecting the surgeon against droplet and aerosol contamination. The lack of droplet contamination on face shields of the surgeon and scrub nurse implied that the plastic sheet was effective in preventing droplet and aerosol spillage.

Results from our preliminary study suggested that the use of 2 horizontal anaesthetic screens and a clear sterile plastic sheet draped over a tracheostomy operative field can effectively prevent droplet contamination, obviating the need for a face shield given adequate eye protection and respirator. Such an approach can also be advocated for other AGP in an attempt to reduce droplet and aerosol contamination, and ultimately viral transmission to health care providers.

Larger scale studies with more patients and operating surgeons is warranted to justify such recommendations. Given the effectiveness of the plastic sheet in preventing droplet contamination, the role and efficacy of N95 respirator versus medical masks in preventing viral transmission can be re-assessed.

\section{CONCLUSION}


The use of 2 horizontal anaesthetic screens and a sheet of clear sterile plastic drape effectively creates a closed sterile environment for the surgeon to perform AGP on all unknown, suspected and confirmed COVID-19 patients, whilst minimising the chance of droplet contamination and viral transmission to health care providers. Such a set-up is functional, readily available and cost effective. PPE such as face shield can be conserved. The aforementioned approach should be considered to support safe clinical practice and efficient use of resources during the COVID-19 pandemic.

\section{REFERENCES}

1. Zou L, Ruan F, Huang M, et al. SARS-CoV-2 Viral Load in Upper Respiratory Specimens of Infected Patients. N Engl J Med. February 2020: doi:10.1056/NEJMc2001737. [Epub ahead of print]

2. US Centers for Disease Control and Prevention. Interim infection prevention and control recommendations for patients with suspected or confirmed coronavirus disease (COVID-19) in healthcare settings. [Available. From: https://www.cdc.gov/coronavirus/2019-ncov/infection-control/controlrecommendations.html]

3.

\section{Tay JK, Khoo ML, Loh WS. Surgical Considerations for Tracheostomy Dur- ing the COVID-19 Pandemic: Lessons Learned From the Severe Acute Respiratory Syndrome Outbreak.JAMA Otolaryngol Head Neck Surg. 2020 Mar 31. doi: 10.1001/jamaoto.2020.0764. [Epub ahead of print]}

Lai C-C, Liu YH, Wang C-Y, et al. Asymptomatic carrier state, acute respiratory disease, and pneumonia due to severe acute respiratory syndrome coronavirus 2 (SARSCoV-2): Facts and myths. J Microbiol Immunol Infect. 2020;2:1-36. doi:10.1016/j.jmii.2020.02.012 Ai T, Yang Z, Hou H, et al. Correlation of Chest CT and RT-PCR Testing in Coronavirus Disease 2019 (COVID-19) in China: A Report of 1014 Cases. Radiology. 2020:200642. doi:10.1148/radiol.2020200642 World Health Organisation (WHO). Rational use of personal protective equipment for coronavirus disease (COVID-19) and considerations during severe shortages. Interim guidance 2020. [Available from: https://apps.who.int/iris/bitstream/handle/10665/331695/WHO2019-nCov-IPC_PPE_use-2020.3-eng.pdf]

\section{Centers for Disease Control and Prevention (CDC) Healthcare In- fection Control Practices Advisory Committee (HICPAC). Part III. Precautions to Prevent Transmission of Infectious Agents. [Avail- able from: http://ww.cdc.gov/hicpac/2007IP/2007ip_part3.html]}

Centre for Health Protection. Key Elements on Prevention and Control of Coronavirus disease (COVID-19) in Healthcare Settings (Interim) . [Available from: https://www.chp.gov.hk/files/pdf/ic_advice_for_nid_in_healthcare_setting.pdf] World Health Organisation (WHO). Novel Coronavirus (COVID-19) Situation Dashboard. [Available from: https://who.sprinklr.com] Centre for Health Protection. Latest Situation of Coronavirus Disease (COVID-19) in Hong Kong. [Available from: https://chp-dashboard.geodata.gov.hk/covid19/en.html] Wei WI, Tuen HH, Ng RW, Lam LK. Safe tracheostomy for patients with severe acute respiratory syndrome. Laryngoscope. 2003;113(10):1777-1779. Vargas M, Servillo G. Improving staff safety during tracheostomy in COVID-19 patients. Head Neck. 2020 Apr 9. doi: 10.1002/hed.26163. [Epub ahead of print] 
Bann DV, Patel VA, Saadi R, et al. Impact of Coronavirus (COVID-19) on Otolaryngologic Surgery: A Brief Commentary. Head Neck. 2020 Apr 9. doi: 10.1002/hed.26162. [Epub ahead of print]

\section{LEGEND FOR FIGURES}

Figure 1. Placement of 2 horizontal anaesthetic screens which were secured by universal rotary clamps on patient's left bedside.

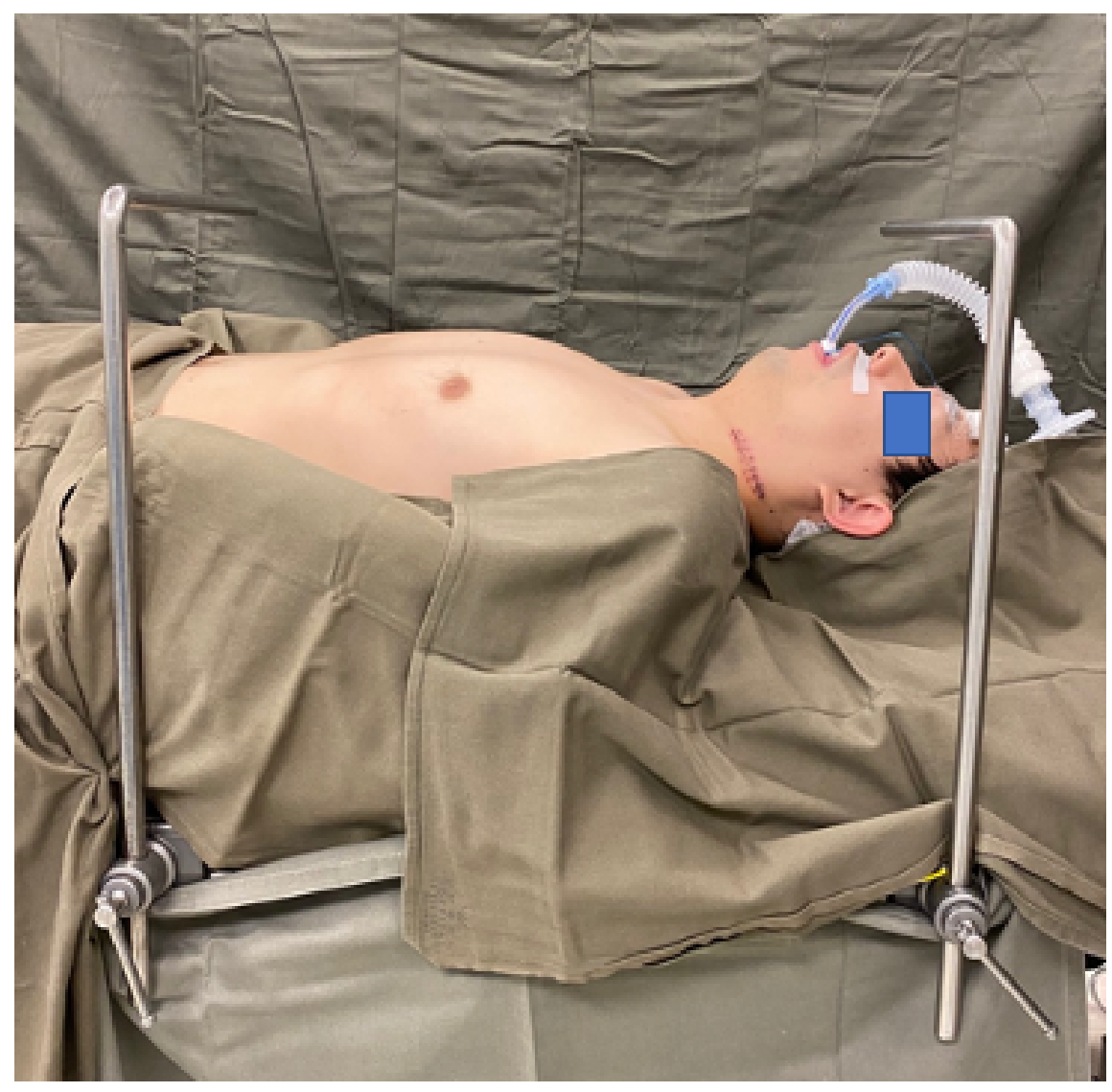

Figure 1: This is a caption

Figure 2. Placement of a piece of taut, clear and sterile plastic sheet over the operative field. The sheet was fixed with sterile clamps on the 2 horizontal anaesthetic screens. The caudal and left lateral edges were sealed with $3 \mathrm{M}$ adhesives. 


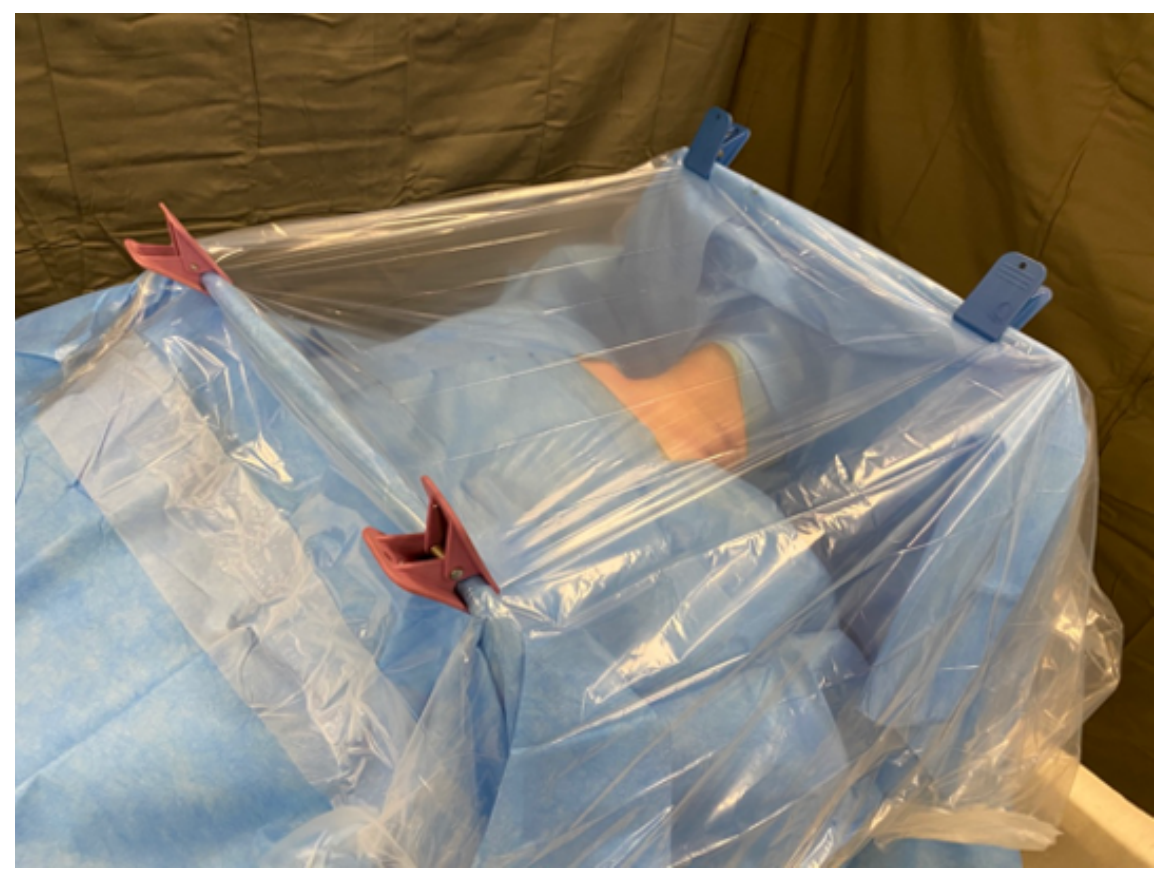

Figure 2: This is a caption

Figure 3. Placement of smoke evacuation suction catheter over the left upper corner.

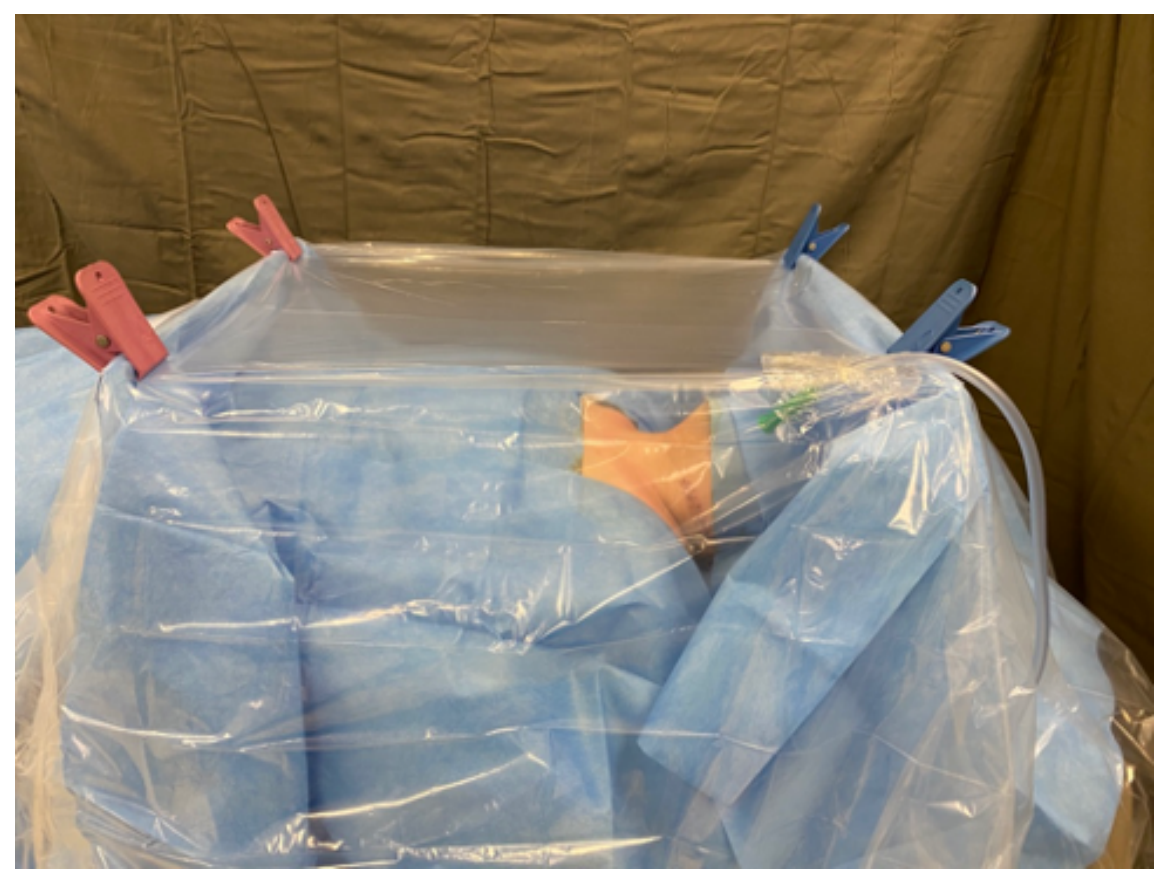

Figure 3: This is a caption 
Figure 4. Placement of grids on plastic sheet on completion of tracheostomy.

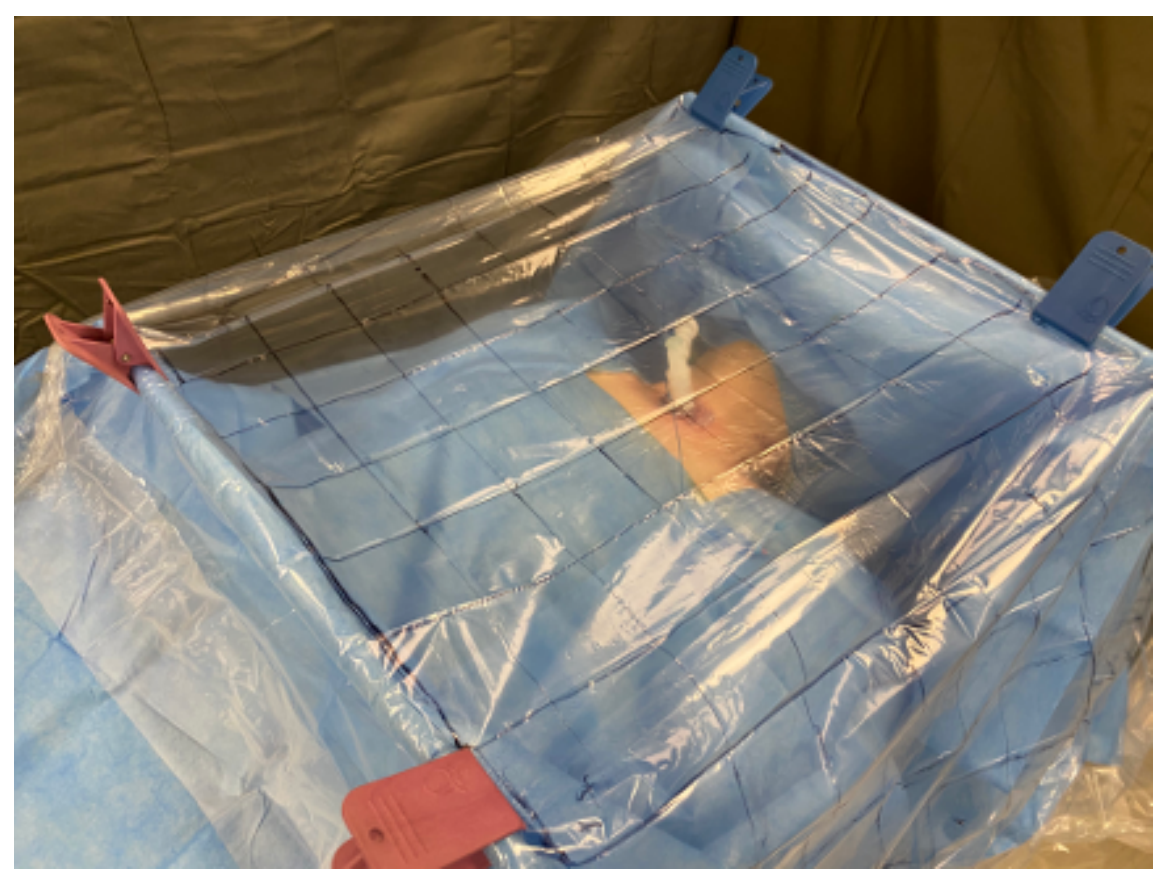

Figure 4: This is a caption

\section{LEGEND FOR TABLE}

\section{Hosted file}

COVID-19_Trach-_(HN)_Table_1.docx available at https://authorea.com/users/312328/articles/444981tracheostomy-during-covid-19-pandemic-a-novel-approach

Table 1. Labelling of grids on plastic drape.

\section{Hosted file}

COVID-19_Trach_(HN)_Table_2.docx available at https://authorea.com/users/312328/articles/444981tracheostomy-during-covid-19-pandemic-a-novel-approach

Table 2. Droplet count and distribution for Patients 1-5.

\section{Hosted file}

COVID-19_Trach_(HN)_-Table_3.docx available at https://authorea.com/users/312328/articles/444981tracheostomy-during-covid-19-pandemic-a-novel-approach

Table 3. Total droplet count and distribution of droplets for Patients 1-5. 